\title{
Ultra-broadband infrared pump-probe spectroscopy using synchrotron radiation and a tuneable pump
}

\author{
Lee Carroll, ${ }^{1}$ Peter Friedli, ${ }^{1}$ Philippe Lerch, ${ }^{2}$ Jörg Schneider, ${ }^{2}$ Daniel Treyer, ${ }^{2}$ \\ Stephan Hunziker, ${ }^{2}$ Stefan Stutz, ${ }^{1}$ and Hans Sigg ${ }^{1}$ \\ ${ }^{1}$ Laboratory for Micro and Nanotechnology, Paul Scherrer Institut, CH-5232 Villigen PSI, Switzerland \\ ${ }^{2}$ Swiss Light Source, Paul Scherrer Institut, CH-5232 Villigen PSI, Switzerland
}

(Received 6 December 2010; accepted 28 April 2011; published online 1 June 2011)

\begin{abstract}
Synchrotron infrared sources have become popular mainly because of their excellent broadband brilliance, which enables spectroscopically resolved spatial-mapping of stationary objects at the diffraction limit. In this article we focus on an often-neglected further advantage of such sources - their unique time-structure - to bring such broadband spectroscopy to the time domain, for studying dynamic phenomenon down to the $100 \mathrm{ps}$ limit. We describe the ultra-broadband (12.5 to $1.1 \mu \mathrm{m}$ ) Fourier transform pump-probe setup, for condensed matter transmission- and reflection-spectroscopy, installed at the X01DC infrared beam-line of the Swiss Light Source (SLS). The optical pump consists of a widely tuneable $100 \mathrm{ps} 1 \mathrm{kHz}$ laser system, covering $94 \%$ of the 16 to $1.1 \mu \mathrm{m}$ range. A thorough description of the system is given, including (i) the vector-modulator providing purely electronic tuning of the pump-probe overlap up to $1 \mathrm{~ms}$ with sub-ps time resolution, (ii) the $500 \mathrm{MHz}$ data acquisition system interfaced with the experimental physics and industrial control system (EPICS) based SLS control system for consecutive pulse sampling, and (iii) the step-scan time-slice Fourier transform scheme for simultaneous recording of the dual-channel pumped, un-pumped, and difference spectra. The typical signal/noise ratio of a single interferogram in a $100 \mathrm{ps}$ time slice is 300 (measured during one single $140 \mathrm{~s}$ TopUp period). This signal/noise ratio is comparable to that of existing gated Globar pump-probe Fourier transform spectroscopy, but brings up to four orders of magnitude better time resolution. To showcase the utility of broadband pump-probe spectroscopy, we investigate a Ge-on-Si material system similar to that in which optically pumped direct-gap lasing was recently reported. We show that the mid-infrared reflection-spectra can be used to determine the optically injected carrier density, while the mid- and near-infrared transmission-spectra can be used to separate the strong pump-induced absorption and inversion processes present at the direct-gap energy. (C) 2011 American Institute of Physics. [doi:10.1063/1.3592332]
\end{abstract}

\section{INTRODUCTION}

The first electron storage ring dedicated to material investigations was commissioned in the 1960s; today, machines of the third generation are being built. ${ }^{1}$ Synchrotron radiation as an infrared (IR) source was already discussed in the 1970s (Ref. 2 and references therein] and the first beamlines dedicated to using the broadband IR from a synchrotron were built in the late 1980s, ${ }^{3-6}$ with spectro-microscopy using synchrotron mid-IR developed in the 1990s. ${ }^{7,8}$ Synchrotron radiation-infrared (SRIR) has two principal advantages over tabletop broadband thermal sources, such as a Globar or Hg-lamp; (i) SRIR has higher-brilliance, and (ii) SRIR has a well-defined sub-nanosecond time-structure. The brilliance advantage of SRIR is now routinely exploited to achieve near diffraction-limited imaging for IR-microscopy/spectroscopy, and a literature-search on this topic will return hundreds of articles (for a review, see Ref. 8). In contrast, there are limited examples of experiments ${ }^{9-11}$ exploiting the fact that each sub-nanosecond pulse of SRIR contains spectrally broadband radiation spanning the full mid-infrared (MIR) and nearinfrared (NIR) range, see Fig. 1(a). Those few experiments using the time-structure of SRIR to perform infrared pumpprobe (IRPP) spectroscopy make use of high repetition-rate lasers as pump sources. In its simplest form, this approach uses a 1:1 matching of the laser system repetition-rate and the clock-rate of the synchrotron source, with fast-scan Fourier transform infrared (FTIR) spectroscopy. Such systems can only operate with their maximum pulse-limited time resolution, if the pump-induced effects have completely recovered before the next pump/probe pulse arrives. ${ }^{10}$ Furthermore, broad tuning of the pump sources into the MIR is not straightforward, because their laser oscillator pulses have insufficient intensity to drive the necessary non-linear optical processes.

Since the Swiss Light Source (SLS) operates at $500 \mathrm{MHz}$, a factor of $\times 5$ times higher than commercially available laser oscillators, the simple 1:1 IRPP scheme of other infrared beam-lines cannot be used. In this article, we describe the newly developed SLS-IRPP system at the X01DC beam-line of the Swiss Light Source, which offers measurements with $100 \mathrm{ps}$ time resolution out to $1 \mathrm{~ms}$ if necessary, and a widely tuneable (16 to $1.1 \mu \mathrm{m}) 100 \mathrm{ps}$ laser system that covers much of the NIR and MIR range as the pump. This new step-scan FTIR SLS-IRPP system, therefore, provides both improved spectral-bandwidth for optical pumping and improved time-bandwidth at maximum temporal resolution, as compared to existing synchrotron IRPP systems. The wide tuning range of the pump is possible, 

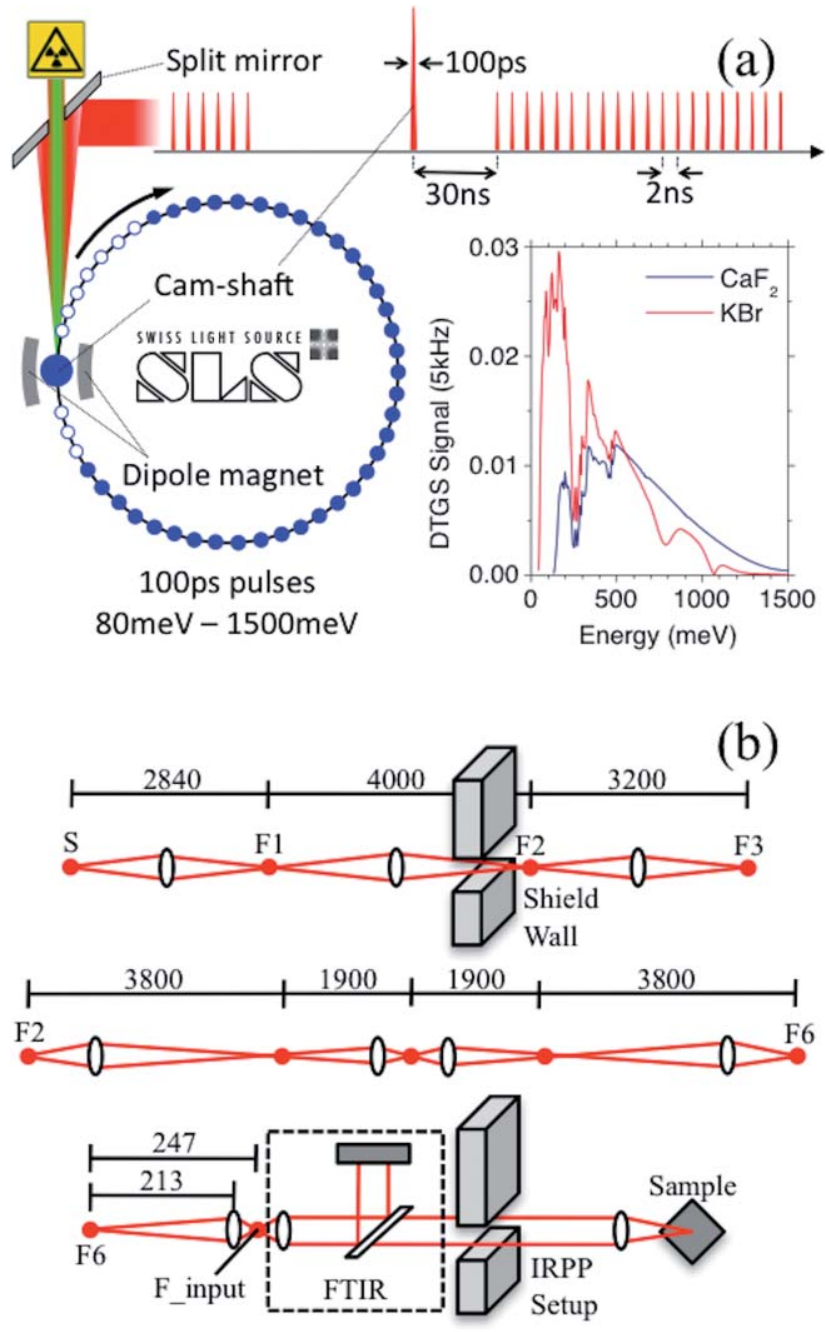

FIG. 1. (Color online) (a) Schematic of the SLS asymmetric filling pattern and SRIR pulse distribution. The inset shows the bandwidth of the broadband SRIR with different FTIR beam-splitters. (b) Schematic of the X01DC beamline. The source (S) is transported along three distinct segments, S-F3, F2-F6, and F6-F7. The telescope optic between F6 and F7 adapts the divergence of the SRIR to the FTIR, and subsequent experimental setup. Both a 1:3.5 and 1:6 demagnification is provided. Near F1, a diamond window separates the machine UHV vacuum from the $10^{-5} \mathrm{mbar}$ of the beam-line. Three conjugate F3 points are available for experiments. A plane mirror (not shown) near F2 can deflect the beam along the transfer-line, which runs from F2 to F6, using toroidal Au-coated mirrors designed to yield 1:1 transport.

because the system uses high-energy pulses delivered by a $1 \mathrm{kHz}$ regenerative amplifier (RegA) to drive several nonlinear optical processes (optical parametric oscillator, amplifier, difference frequency generation, and second harmonic generation). Additionally, the $1 \mathrm{kHz}$ repetition-rate allows material systems with long recovery times, such as those found in photo-activated oxidation/reduction processes mediated by catalytic intermediates ${ }^{12}$ or biological systems, ${ }^{13,14}$ to be followed from the sub-nanosecond to many-microsecond regimes. To utilize this large timebandwidth, a $500 \mathrm{MHz}$ data acquisition system (DAQS) is developed to capture and process individual SRIR signals from pumped and un-pumped events. For maximum temporal resolution, the DAQS is operated in a "camshaft only" mode that offers 100 ps time resolution with a pump-probe offset of up to $1 \mathrm{~ms}$. Optionally, the DAQS allows recoding of two channels simultaneously, which is useful for performing correlated transmission and reflection studies or as a normalization channel. The number of photons in single 100 ps camshaft pulse delivered to the sample is comparable to that from a Globar source integrated over $1 \mu \mathrm{s}$. This comes from the factor of $\times 100$ brilliance advantage of a typical SRIR source such as the SLS, and the $4 \mathrm{~mA}$ current concentrated in the camshaft pulse. In other words, our IRPP system offers similar signal/noise $(\mathrm{S} / \mathrm{N})$ ratio to measurements made with a Globar, but at up to 4 orders of magnitude better time resolution. If long -lived effects are under investigation, then improved $\mathrm{S} / \mathrm{N}$ is achieved by using many (10, 100 or more) of the closely $500 \mathrm{MHz}$ SRIR pulses as the probe signal, instead of just the camshaft pulse. In this case, the DAQS operates as a flexible gate and boxcar integrator giving adjustably long time slices (for example $\sim 0.1 \mu \mathrm{s}$ ) at time delays of $>1 \mu \mathrm{s}$. This box-car mode is thus similar to the widely used time resolved (TR) step-scan FTIR technique developed for study of photo-flash induced chemical reaction paths at sub- $\mu$ s down to 10 ns time scales. ${ }^{13-17}$ At longer times scales, when the detector signal is integrated over several of the $500 \mathrm{MHz}$ pulses, the advantage of our system over the Globar source is the higher brilliance $(\sim 100 \mathrm{x})$ of the synchrotron source. This is useful for, experiments with small samples, when using fast, small-area detectors, and investigation with geometrical constrains, i.e., low temperature cryostats, pressure cells, and photo catalyzes studies made at grazing-incidence. This review deals mainly with the novel aspects of our "camshaft only" measurements, which probe the sub- and multiple-ns time regime that could not previously be accessed by TR step-scan FTIR methods (because it is too fast), or femto-second laser based TR spectroscopy (because of delay-line limitations).

This article is organized as follows - in Sec. II we describe the SRIR at the X01DC beam-line of the SLS, the synchronization of the laser system to the SRIR pulses, the optical layout of the experiment, and the DAQS system used to interface with the Fourier transform IR spectrometer. In Sec. III we discuss the noise and stability of the SRIR pulses and the noise of the IRPP interferograms. In Sec. IV we present broadband IRPP transmission- and reflection-spectroscopy of a Ge-on-Si material system, motivated by recent reports of optically pumped Ge-on-Si lasing. ${ }^{18}$ We show (i) the reflection-spectra give insight into the dynamics of the injected carrier density, and (ii) the transmissionspectra reveal the competition between strong pump-induced absorption and inversion at the direct gap energy.

\section{EXPERIMENTAL}

\section{A. Synchrotron-infrared at the SLS}

The SLS is a third generation synchrotron light source. It operates in a TopUp mode that keeps the total beam-current between $400 \mathrm{~mA}$ and $402 \mathrm{~mA}$ by periodically $(\approx 140 \mathrm{~s})$ injecting electrons into the orbiting electron bunches. A pulse of synchrotron radiation is generated every time an electron bunch passes through the $1.4 \mathrm{~T}$ continuous-field dipole 
magnet of the X01DC infrared beam-line. ${ }^{19} \mathrm{~A}$ water-cooled plane-mirror with a $2 \mathrm{~mm}$ slit, placed $0.8 \mathrm{~m}$ from the source (S), out-couples the SRIR, but "passes" the UV and x-ray radiation. Figure 1(b) sketches the beam-path from $S$ to the F7 of the Bruker Vertex-70 FTIR. An elliptical mirror matches the transported SRIR to the acceptance angle of the dry-air purged FTIR. The full-bandwidth (visible to mid-IR) average power at F2 and F6 is $12 \mathrm{~mW}$ and $5.8 \mathrm{~mW}$, respectively. The total FTIR-modulated SRIR power delivered to the IRPP setup is $500 \mu \mathrm{W}$ to $1 \mathrm{~mW}$, depending on the telescope optic between F6 and F7. This power is significantly less than at F6, because of beam-splitter losses and beam clipping.

During IRPP measurements, the SLS operates with an asymmetric filling pattern, consisting of 480 electron "buckets," of which 89 are empty, 390 are filled with standard electron-bunches, and 1 is filled with the camshaft bunch (see Fig. 1(a)). The standard bunch separation is $2 \mathrm{~ns}$, giving the SRIR a nominal $500 \mathrm{MHz}$ repetition-rate. The single camshaft contains four times the number of electrons as a standard bunch and has a repetition-rate of $1 \mathrm{MHz}$. It is provided for users making time-resolved measurements. Since it has a $30 \mathrm{~ns} / 150 \mathrm{~ns}$ offset from the next/previous standard bunch, the $100 \mathrm{ps}$ camshaft pulse can be resolved with a $30 \mathrm{MHz}$ detector/amplifier system. Focussed with a $100 \mathrm{~mm}$ parabolic mirror, 95\% of the SRIR $(\lambda>1 \mu \mathrm{m})$ passes through a $200 \mu \mathrm{m}$ pinhole. This is more than a factor of $\times 20$ over the internal $250 \mu \mathrm{m}$-apertured Globar source of the FTIR $(30 \mu \mathrm{W}) .{ }^{20}$ Furthermore, the camshaft has a 100ps peak-intensity advantage of more than $\times 2000$ over the Globar. Using a $30 \mathrm{MHz}$ bandwidth detector with a Globar source yields a time resolution of tens of nanoseconds, but with the camshaft a true $100 \mathrm{ps}$ resolution can be achieved, while profiting from the moderate detection-noise bandwidth.

It should be noted, however, that while a Globar benefits from extreme stability, SRIR pulses vary in intensity because of TopUp pointing stability affected by storage-ring orbital feedback systems and mechanical vibrations. These factors act to erode some part of the IRPP signal advantage and so limit the achievable spectral $\mathrm{S} / \mathrm{N}$ ratio.

\section{B. Synchronized pump system}

Based on designs from the laser group of Professor Zacharias at the Westfälische Wilhelms Univestity of Münster, ${ }^{21-23}$ the $100 \mathrm{ps} 1 \mathrm{kHz}$ pump source covers $94 \%$ of the 1.1-16 $\mu \mathrm{m}$ tuning range, see Fig. 2(a). This pumping range is considerably broader than that offered at other synchrotron IRPP systems and offers users maximum flexibility. The system uses a $1064 \mathrm{~nm} \mathrm{Nd:YAG} \mathrm{regenerative} \mathrm{amplifica-}$ tion, a quasi-continuous wave $(\mathrm{CW})$ pumped optic parametric oscillation (OPO), optic parametric amplification (OPA), difference frequency generation (DFG), and second harmonic generation (SHG) stages to cover this wide tuning range. Details of the system can be found in the Appendix. The missing $6 \%$ in the tuning range corresponds to the dead-zone in OPO/OPA performance around the $1064 \mathrm{~nm}$ conjugate-point $(2.02-2.25 \mu \mathrm{m})$. The system was custom manufactured by Ekspla, and includes a piezo-controlled master-oscillator
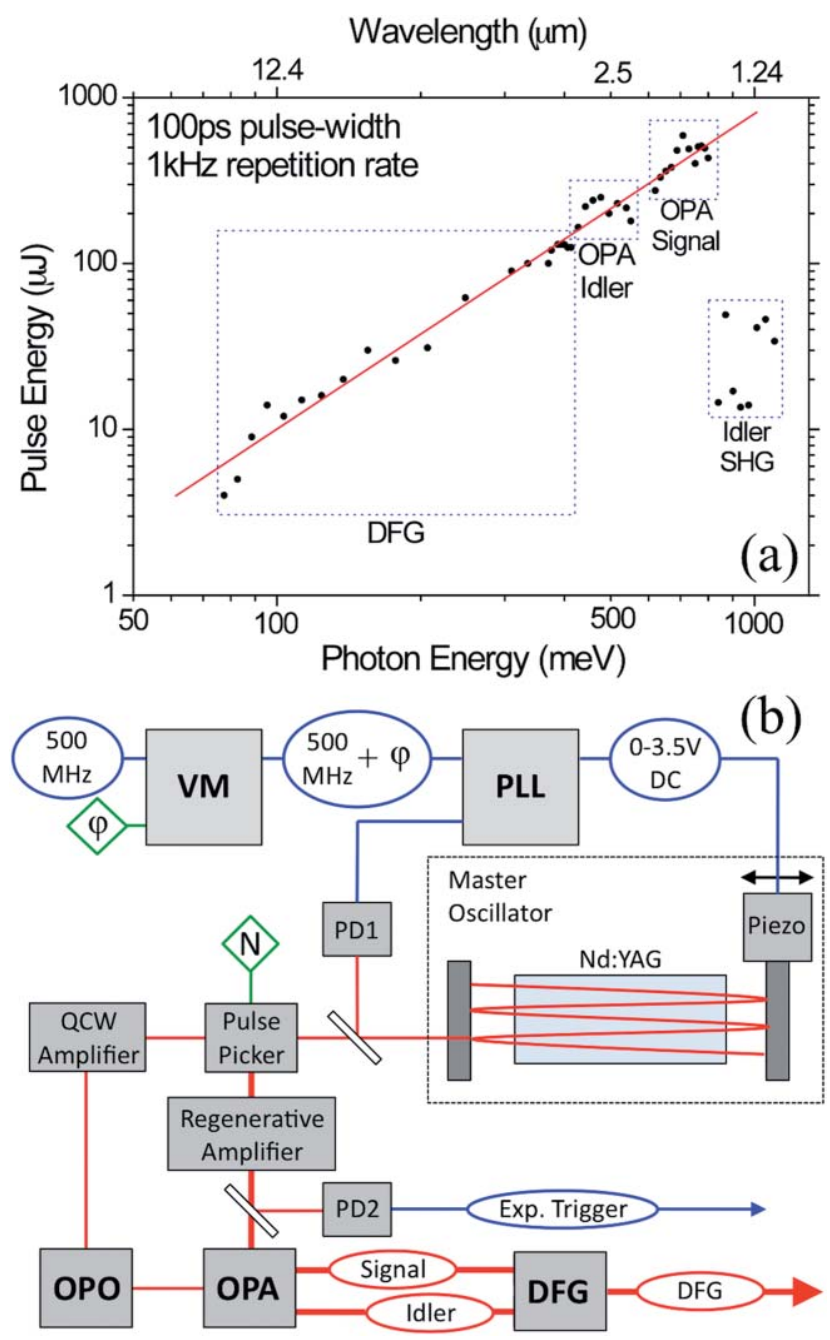

FIG. 2. (Color online) (a) Pump pulse-energy (single shot) vs. photon energy/wavelength for the tuneable pump-source. The DFG, OPA, and SHG tuning ranges are indicated. (b) Optical beam-path of pump-source when configured for DFG pulses. The schematic includes selected electronic/control lines used to achieve synchronization to the SLS.

(MO) cavity, to enable synchronization with the sixth subharmonic of the SLS clock-rate $(83.3 \mathrm{MHz}=500 \mathrm{MHz} / 6)$. A digital pulse-picker selects an MO pulse that is integer multiple of the 480 SLS buckets (83520) for regenerative amplification.

A $100 \mathrm{~Hz}$ bandwidth phase-lock-loop (PLL) system is used to synchronize the MO to the SLS. The PLL system routinely maintains a lock over several days of beam-time operation. Integrated phase-noise measurements $(1 \mathrm{kHz}$ to $10 \mathrm{MHz}$ ), made with an Agilent 5052B signal source analyzer, gives a MO jitter of $200 \mathrm{fs}$ and $400 \mathrm{fs}$ in free running and PLL operation, respectively. When the PLL has locked the MO to the SLS clock-rate, all $1 \mathrm{kHz}$ pump pulses (OPA, DFG, and SHG) are locked to the SRIR camshaft pulses. Since the initial lock occurs at some arbitrary phase, see Fig. 3(a), an offset of up to $500 \mathrm{~ns}$ between the pump and nearest camshaft pulse exists. Once lock has been achieved it is very stable - we have never observed the system "slip" to another bucket, even when operated over several days of beam-time. The maximum jitter of the camshaft bunch, with respect to the $500 \mathrm{MHz}$ 
clock-rate is $<10 \mathrm{ps}$, fully one order of magnitude less than the $100 \mathrm{ps}$ maximum resolution.

An $\mathrm{RF}$ vector-modulator (VM) is used to introduce a phase-shift on the $500 \mathrm{MHz}$ SLS clock-rate fed to the input of the PLL. With the correct phase-shift, the pump and camshaft pulse are brought into coincidence, see Fig. 2(b). The $500 \mathrm{fs}$ $\left(2^{12} \times 500 \mathrm{MHz}\right)$ resolution of the VM is far better than the $100 \mathrm{ps}$ pump pulse-width, and there is no practical limit to the pump-probe offset it can introduce. The VM eliminates the need for a physical optical delay line and offers fully digital control of offsets from $500 \mathrm{fs}$ to $1 \mathrm{~ms}$. In its sweep-mode, the VM can drive the PLL with a maximum phase-shift velocity of $160 \mathrm{rad} \mathrm{s}^{-1}(100 \mathrm{~Hz} \times \pi / 2)$. This gives a maximum system slew-rate of $50 \mathrm{~ns} \mathrm{~s}^{-1}$, so first-order temporal overlap between the pump and nearest camshaft pulse takes only tens of seconds.

\section{Optical layout}

Since the pump source operates with a low duty-cycle, the IRPP FTIR operates in step-scan mode - moving the mirror a discrete step, holding that position while the system integrates over a given number of laser-shots (typically 85), and then moving to the next step, etc. For the measurements made in Sec. IV, the FTIR-modulated SRIR is delivered to the IRPP setup as a near-collimated beam, and focussed to the sample using an $\mathrm{f}=100 \mathrm{~mm} 90^{\circ} \mathrm{Au}$-coated parabolic mirror. Apart from the FTIR beam-splitter and two vacuum windows, all-metal optics are used throughout in order to maximize broadband throughput and minimize dispersive effects. As mentioned previously, the SRIR $(\lambda>1 \mu \mathrm{m})$ is focussed to better than $200 \mu \mathrm{m}$ in the sample-plane. Both transmitted and reflected SRIR are collected and focussed to suitable detectors using additional parabolic mirrors.

The detector choice depends on the spectral range under investigation. The specifications of the four fast detector systems available are given in Table I. Because of the very large difference in pump and probe powers, filtering of the pump from the detectors is necessary to exclude background signals. Pump signal "leaking" onto a detector is not FTIR modulated and so does not generate a spectral artefact directly, but it may act as a source of noise, or drive the detector into non-linearity. Similarly, un-modulated photoluminescence from a pumped sample has been observed to act as a significant source of noise.

For the Ge measurements presented in Sec. IV, the $1064 \mathrm{~nm}$ RegA pump was focussed to the sample plane with an $\mathrm{f}=300 \mathrm{~mm}$ BK7 plano-concave lens. First-order spatial overlap of the pump and probe was tested using a
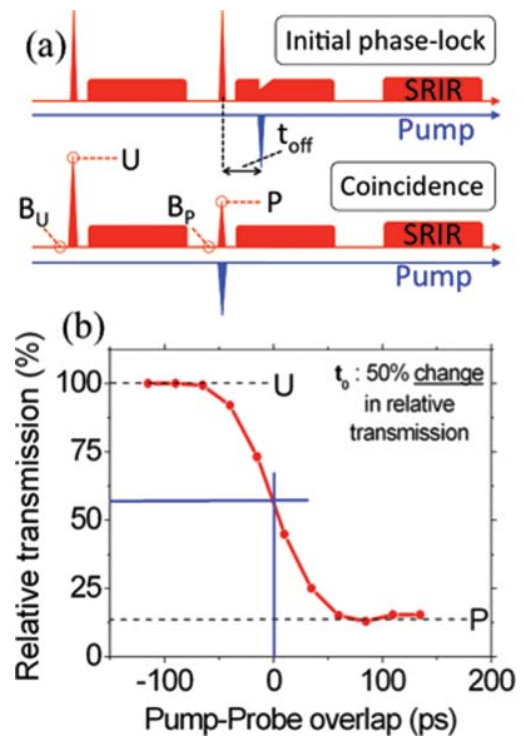

FIG. 3. (Color online) (a) Schematic of the SRIR pulse-train transmitted through a pumped sample. In the initial arbitrary phase-lock, the pump-probe offset $\left(\mathrm{t}_{\mathrm{off}}\right)$ is up to $500 \mathrm{~ns}$. After temporal overlap is achieved, the difference between the un-pumped $(\mathrm{U})$ and pumped $(\mathrm{P})$ camshaft signals is used to generate the IRPP spectra. (b) Relative transmission of a Ge optical-flat when pump-probe overlap is achieved. Data taken from the experiment described in Sec. IV. Experimental "time-zero" $\left(\mathrm{t}_{0}\right)$ is taken to be the moment when the change in relative transmission is $50 \%$ of its maximum.

retractable $200 \mu \mathrm{m}$ pinhole inserted into the sample-plane. Detuning the focal point of the pump, such that $\approx 50 \%$ is transmitted through the same pinhole ensured the pump overfilled the SRIR probe spot. Of course, spatial overlap of the pump and probe beams is only the first step - temporal overlap between the pump and probe pulses is also needed.

First-order temporal overlap is achieved by sweeping the VM until the fast $(<1 \mathrm{~ns}) 1 \mathrm{kHz}$ RegA trigger from the laser system overlaps with the nearest camshaft pulse. After allowing for cabling and time-of-flight delays, this estimate can be as good as $1 \mathrm{~ns}$. When the spatial and temporal overlap is optimized, a strong change in the amplitude of the pumped transmitted (and/or reflected) camshaft pulse can usually be observed, see Fig. 3(b). The 50\% value of the pump-induced rising edge $(\approx 100 \mathrm{ps})$ is used to define the zero-time $\left(t_{0}\right)$ for the experiment.

\section{Data Acquisition and Step-Scan FTIR}

When synchronized to the laser, only $0.1 \%(1 \mathrm{kHz} / 1$ $\mathrm{MHz}$ ) of the camshaft pulses passing through the sample are actually pumped. A DAQS is used to systematically sam-

TABLE I. Specifications of fast detectors available for IRPP measurements, including the bandwidth, spectral range, and manufacturer-specified noiseequivalent power (NEP), or specific-detectivity (D*)

\begin{tabular}{|c|c|c|c|}
\hline Detector & Bandwidth & Range (50\% max) & Noise specification \\
\hline Vigo-MCT (TEC-cooled) & $500 \mathrm{MHz}(\mathrm{ac})$ & $10-3.2 \mu \mathrm{m}$ & $\mathrm{D}^{*}=5 \times 10^{9} \mathrm{~cm} \sqrt{ } \mathrm{Hz} / \mathrm{W} @ 500 \mathrm{MHz}$ \\
\hline Teledyne-Judson-InSb ( $\mathrm{LN}_{2}$-cooled) & $200 \mathrm{MHz}(\mathrm{ac})$ & $4.1-1.0 \mu \mathrm{m}$ & $\mathrm{NEP}=1 \mathrm{pW} / \sqrt{ } \mathrm{Hz} @ 1 \mathrm{MHz}$ \\
\hline Femto-InGaAs & $400 \mathrm{MHz}(\mathrm{ac})$ & $1.7-1.0 \mu \mathrm{m}$ & $\mathrm{NEP}=21 \mathrm{pW} / \sqrt{ } \mathrm{Hz} @ 100 \mathrm{MHz}$ \\
\hline Kolmar-MCT ( $\mathrm{LN}_{2}$-cooled) & $50 \mathrm{MHz}(\mathrm{dc})$ & $11-1.4 \mu \mathrm{m}$ & $\mathrm{D}^{*}=4 \times 10^{10} \mathrm{~cm} \sqrt{ } \mathrm{Hz} / \mathrm{W} @ 50 \mathrm{kHz}$ \\
\hline
\end{tabular}




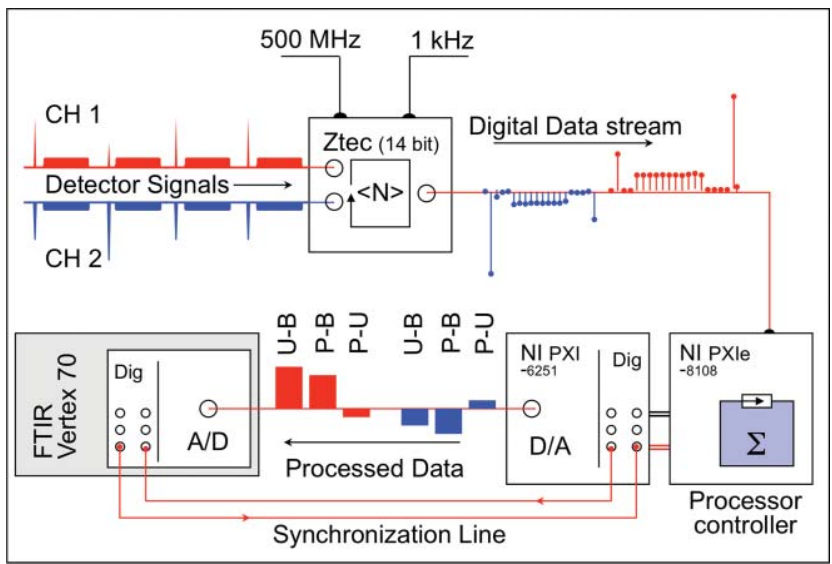

FIG. 4. (Color online) Schematic of dual-channel mode DAQS operation. The two detector channels are simultaneously sampled by the ZTEC, which is triggered by the EPICS. The digitized data (full $1 \mu$ s time-bandwidth) is sent to process-controller for averaging, peak-picking and background subtraction. The six measurement values are then sent to the FTIR analog-to-digita (A/D) with synchronization provided for mirror-step delays, sampling, etc.

ple the pumped and (immediately preceding) un-pumped camshaft pulses. The system is based on a National Instruments (NI) PXIe-1062Q chassis, with a controller (NI PXIe8108RT) running a real-time operating system. To input signal from the fast detectors, we use a 14-bit, $250 \mathrm{MHz}$ bandwidth PXI digitizer module (ZTEC ZT410-21), and a 16-bit, 1.25 MS/s multi-channel module (NI PXI-6251DAQ). Using the SLS $500 \mathrm{MHz}$ clock-rate to bypass the internal PXI clock ensures reproducible, synchronous sampling of the camshaft pulses.

Usually, the DAQS operates in a dual-channel mode, typically using one channel each for reflection and transmission measurements. The DAQS uses a $1 \mu$ s burst of high frequency sampling per laser shot to capture the detector response. In dual-channel mode, the burst sampling-rate and bandwidth are $250 \mathrm{MHz}$. The burst is locked to the laser system via a $1 \mathrm{kHz}$ trigger signal derived from the Micro-Research VMEEVR-230RF EPICS event receiver system at the SLS. The phase of the $1 \mathrm{kHz}$ EPICS trigger can be electronically shifted in steps of $20 \mathrm{~ns}$, to give a first-order overlap between the sampling burst and the pumped camshaft. The DAQS itself provides further tuning in steps of $2 \mathrm{~ns}$, based on the SLS $500 \mathrm{MHz}$. For detectors with bandwidth $>250 \mathrm{MHz}$, finetuning with an analogue phase-shifter is necessary to ensure DAQS sampling on the peak detector response.

On receiving the $1 \mathrm{kHz}$ EPICS trigger, the DAQS acquires both detector signals for a full $1 \mu \mathrm{s}$, and then uses a real-time LABVIEW routine to identify four values on each channel: un-pumped background $\left(\mathrm{B}_{\mathrm{U}}\right)$, un-pumped camshaft signal $(\mathrm{U})$, pumped background $\left(\mathrm{B}_{\mathrm{P}}\right)$, and pumped camshaft signal (P), see Fig. 3(a). For slow detectors (bandwidth $<100 \mathrm{MHz}$ ), the DAQS can integrate the detector pulse over several points to improve the measurement quality. The DAQS calculates the values of $[\mathrm{P}-\mathrm{U}],\left[\mathrm{U}-\mathrm{B}_{\mathrm{U}}\right]$, and $\left[\mathrm{P}-\mathrm{B}_{\mathrm{P}}\right]$ for both channels and averages the six measurements over the user-specified number of laser shots (typically 85 ). The averaging is synchronized to the step-scan of the FTIR, which sends a step trigger to the PXI, when the mirror position

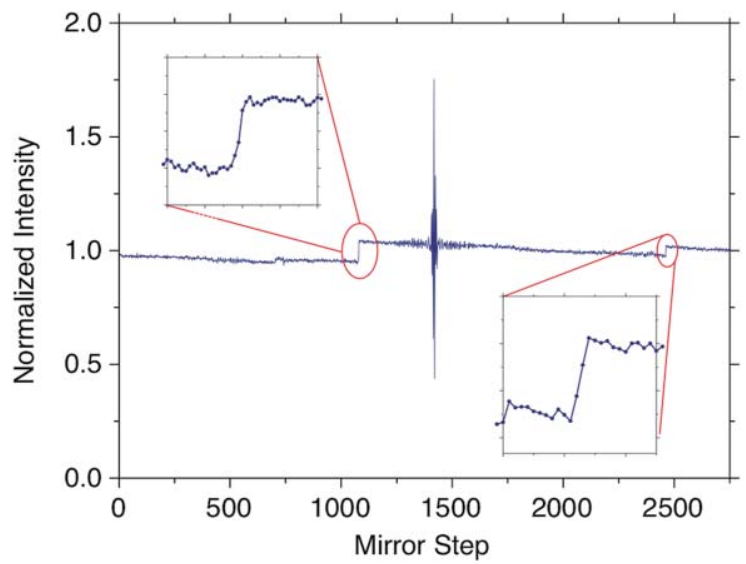

FIG. 5. (Color online) Single scan $\left[\mathrm{U}-\mathrm{B}_{\mathrm{U}}\right]$ interferogram, measured through two TopUp events, and so including offset artefacts. The first TopUp feature is due to a "double" injection, increasing the camshaft intensity by $7 \%$. The more typical, single injection introduces a $3.5 \%$ step.

moves. After the averaging, the DAQS sends a read-in trigger to the external trigger box of the FTIR (E525/Z), and after a short delay, sends a six-level analogue voltage-train to the FTIR ADC, see Fig. 4. The FTIR samples its analogue to digital converter (ADC) with six time-slices, recording the value of $[\mathrm{P}-\mathrm{U}]_{1},\left[\mathrm{U}-\mathrm{B}_{\mathrm{U}}\right]_{1},\left[\mathrm{P}-\mathrm{B}_{\mathrm{P}}\right]_{1}$, etc., as the interferogram amplitudes of that mirror position. The FTIR then moves to the next mirror position and the process is repeated.

The total number of mirror-steps per scan is determined by the range and resolution of the spectrum, while the total integration time is limited by the SLS TopUp period for the camshaft, which is presently $140 \mathrm{~s}$. Integrating across a TopUp event should be avoided because it introduces a stepfeature in the interferogram, see Fig. 5. The origin of this step is the $3.5 \%$ or $7 \%$ change in camshaft intensity during TopUp. This change is so large, because the camshaft lifetime is significantly less than that of the standard bunches, due to its factor of $\times 4$ higher electron density. A C\# application is used to synchronize the FTIR to the EPICS TopUp trigger ensuring that the scan starts immediately after the TopUp event, and has the full $140 \mathrm{~s}$ period. This application is also used to perform measurement co-additions, and is also interfaced with the VM to facilitate programmable control of the pump-probe time offset. Efforts are underway to normalize the FTIR signal to the camshaft current (which is provided as an EPICS channel), thereby allowing long scans to be made through multiple TopUp events.

Because of the mirror-move/stabilization $(16 \mathrm{~ms} / 5 \mathrm{~ms})$, DAQS processing $(7 \mathrm{~ms})$, and triggering/signal-transfer delays $(4 \mathrm{~ms} / 6 \mathrm{~ms})$, there is a $38 \mathrm{~ms}$ overhead per mirror-step, regardless of the number of integrated laser-shots. The ratio of the $140 \mathrm{~s}$ TopUp to the $38 \mathrm{~ms}$ overhead gives the maximum achievable IRPP resolution: $15 \mathrm{~cm}^{-1}$ over the full $0-15800 \mathrm{~cm}^{-1}$ range. Typically, the IRPP setup operates with 85 laser shots, giving a $15-30 \%$ dead time and a resolution of $25-100 \mathrm{~cm}^{-1}$

The DAQS can also be used in a single-channel mode, where it captures all camshaft pulses (1 pumped and 1039 un-pumped) per laser-shot from a single detector. A fast 
latch/hold scheme, based on an AnaPico AP3501 sampler and a Micro-Research 4CHTIM-200 timer-board is used. Averaging over the many un-pumped pulses offers improved $\mathrm{S} / \mathrm{N}$ on the reference channel. However, as both the $1 \mathrm{kHz}$ pumped spectra and $1 \mathrm{MHz}$ un-pumped spectra are used to calculate the normalized spectra, the improvement in single-channel $\mathrm{S} / \mathrm{N}$ is only a factor of $\sqrt{ } 2$. In this article, the single-channel mode is used not for IRPP measurements, but rather as a very direct means of measuring the camshaft stability and noisespectra.

\section{MEASUREMENT QUALITY}

The emission from a Globar source is extremely stable, because it benefits from a large thermal inertia and a fixed-mounting inside the FTIR. Repeated co-additions of the Globar interferograms can lead to a near-Poissonian increase of the $\mathrm{S} / \mathrm{N}$ until the detector limit is reached. This is not true for SRIR interferograms, because the synchrotron source has significant noise, introduced by the storage-ring orbital feedback, and mechanical vibrations of the machine elements and transfer optics.

Using the Vigo-MCT to measure the intensity of $\approx 10^{7}$ consecutive SRIR cam-shaft pulses $(\approx 10 \mathrm{~s})$ with the DAQS in single-channel mode, and then performing a fast-Fourier
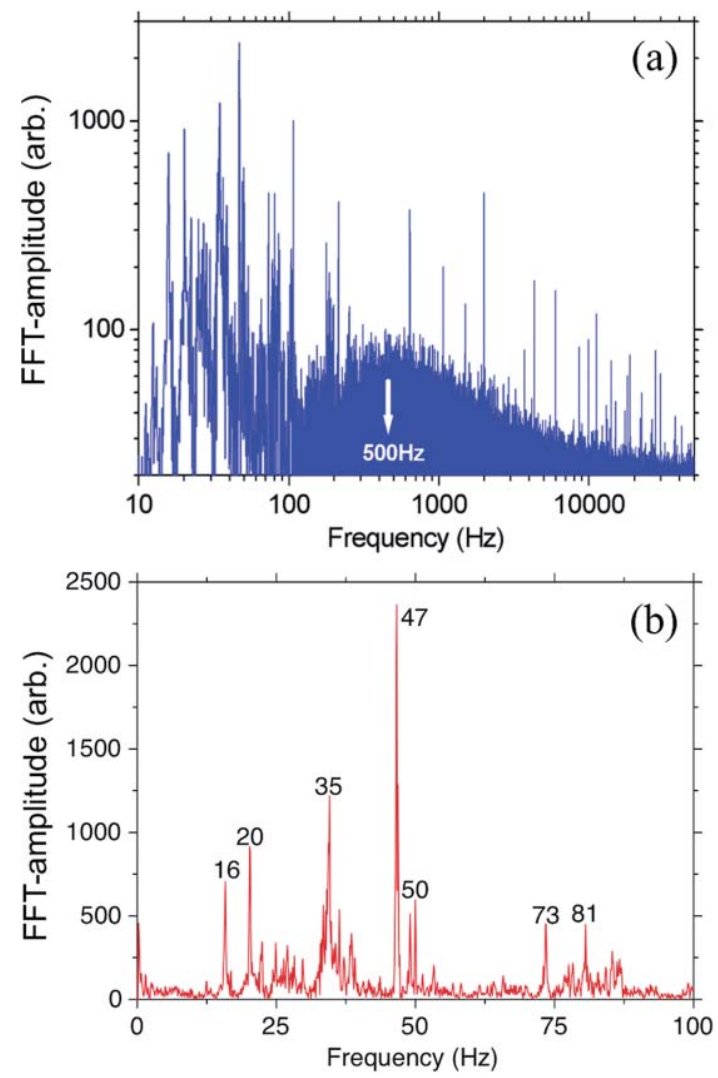

FIG. 6. (Color online) (a) Fast-Fourier transform (FFT) of $10^{7}$ consecutive camshaft pulses captured by the Vigo-MCT, using the single-channel mode DAQS, giving the noise-spectrum of the camshaft pulses. The continuum centred at $500 \mathrm{~Hz}$ is an artefact of the detector amplifier. (b) Detail of the low frequency SRIR noise - correlation measurements with seismic sensor data confirms a mechanical origin for most of the features.

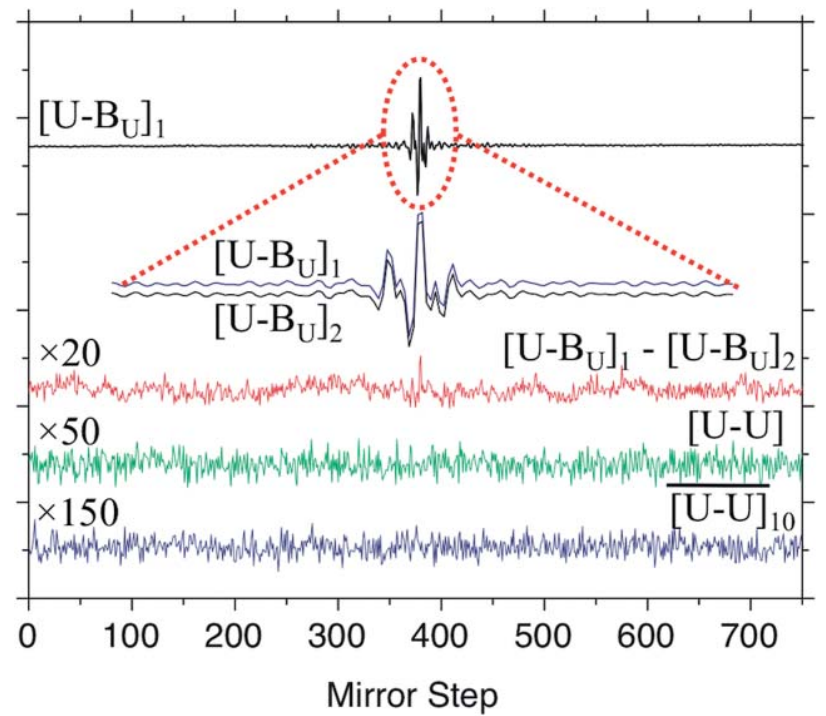

FIG. 7. (Color online) Examples of the [U-B $\left.\mathrm{B}_{\mathrm{U}}\right]$ un-pumped interferograms recorded by the IRPP system, using the Vigo-MCT. Subtracting two measurements made a few minutes apart gives the scan-to-scan stability, which is better than $1 \%$. The RMS noise of a single [U1-U2] scan gives the system $\mathrm{S} / \mathrm{N}=300$. After averaging 10 scans, the RMS noise of [U1-U2 $]_{10}$ gives a typical measurement $\mathrm{S} / \mathrm{N}=900$.

transform, gives the noise spectrum of the full IRPP setup. The noise shown in Fig. 6(a) is dominated by a broad continuum centred on $500 \mathrm{~Hz}$, which originates in the customized trans-impedance amplifier of the Vigo-MCT. The spikes above $1 \mathrm{kHz}$ are thought to RF noise originating from the injection electronics. The low frequency noise, shown in Fig. 6(b) mainly arises from mechanical vibrations of the SLS storagering and the beam-line transfer optics. This has been confirmed by correlation measurements using seismic sensors. The relaxation times of such vibrations, as deduced from the line-widths, are of the order of $1 \mathrm{~s}$. The high frequency $(>100 \mathrm{~Hz})$ noise is efficiently removed from the interferograms by averaging over the 85 laser-shots per mirror-step, but the low frequency SRIR noise will enter the interferograms because the $100 \mathrm{~ms}$ integration-window averages over only a few periods.

The $500-1 \mathrm{MHz}$ bandwidth RMS noise of the $250 \mu \mathrm{m}$ $500 \mathrm{MHz}$ Vigo-MCT without SRIR was measured as $3 \mathrm{mV}$. This is in almost perfect agreement with the theoretical detector-limit calculated from the specifications of $\mathrm{D}^{*}=5$ $\times 10^{9} \mathrm{~cm} \sqrt{ } \mathrm{Hz} / \mathrm{W}$ and $\mathrm{S}=3 \times 10^{4} \mathrm{~V} / \mathrm{W}$. With a camshaft signal of $250 \mathrm{mV}$ (corresponding to an average SRIR intensity of about $4 \mu \mathrm{W}$ ), the measured RMS noise is $5 \mathrm{mV}$. This gives the SRIR RMS noise of $4 \mathrm{mV}$, and a S/N of $\sim 60$. In an IRPP measurement, with the camshaft signal averaged over 85 laser-shots, the total $\mathrm{S} / \mathrm{N}$ should be $550(60 \times \sqrt{ } 85)$ in the Poissonian limit. The real value of the total $\mathrm{S} / \mathrm{N}$ under these conditions was determined by performing a typical dualchannel DAQS measurement without pumping. Here, the [U-P] value reduces to [U1-U2], being the variation in two consecutive un-pumped camshafts (U1 and U2), separated by the $1 \mu$ s revolution time. The RMS noise of [U1-U2], normalized to the amplitude of the $\left[\mathrm{U}-\mathrm{B}_{\mathrm{U}}\right]$ interferogram, gives the total $\mathrm{S} / \mathrm{N}$ of 300 , see Fig. 7. The missing factor of $\times 2$ (from 
550) may be the evidence of the low frequency SRIR noise entering the interferogram. Figure 7 also includes a comparison of two $\left[\mathrm{U}-\mathrm{B}_{\mathrm{U}}\right]$ interferograms, measured a few minutes apart, to illustrate the long-term stability of the SRIR. This is determined to be better than $1 \%$. After performing $10 \mathrm{co}-$ additions the $\mathrm{S} / \mathrm{N}$ is increased to 900 , consistent with the $\sqrt{ } 10$ Poissonian enhancement. However, since each co-addition takes $140 \mathrm{~s}$, the total measurement time was $25 \mathrm{~min}$. Jitter on the pump-probe overlap is a negligible source of additional noise, as determined by comparing the noise on [P-B] signal on the rising edge of the overlap to that on the [U-B] signal.

Future steps to improve system performance may include (i) improved coupling scheme for the FTIR to maximize SRIR throughput to IRPP setup, (ii) active vibration control of the beam using acousto-optic feedback to reduce the low frequency mechanical noise on the SRIR, ${ }^{24}$ and (iii) extension of the camshaft TopUp period.

\section{RESULTS AND DISCUSSION}

The recent report of an optically pumped Ge-on-Si laser from the MIT Microphotonics Center (Cambridge, MA) (Ref. 18) is motivating renewed interest in the physics behind optical-injection and population inversion in Ge. Calculations indicate that a very high optically injected carrier-density $\left(10^{20} \mathrm{~cm}^{-3}\right)$ is needed to achieve net gain. ${ }^{25}$ For such high carrier densities, pump-induced absorption (PIA) is expected to be appreciable, and any net gain will be the result of "competition" between inversion at the direct gap and these absorption channels. Since it spans the full NIR and MIR spectroscopic ranges, the IRPP system is ideally placed to make a contribution to these investigations. The Ge crystals used for bulk investigations are commercially available (uncoated optical-flats from Eksma, with flat transmission from $2-10 \mu \mathrm{m}$ ), and so serve as a performance calibration for the IRPP set-up

\section{A. IRPP investigation of bulk Ge}

It has been known since the 1950s that charge carriers in a semiconductor have a characteristic plasma frequency determined by their density and effective mass. In normal incidence, the plasma frequency typically manifests as a sudden drop in the IR reflectivity with the energy and sharpness of that drop being dependent on the carrier density and scattering-rate, respectively. ${ }^{26}$ With the IRPP system, we optically inject carriers across the Ge direct gap, and follow the plasma-frequency and scattering-rate of the injected-carriers as a function of time. This gives information on the density and dynamics of the carrier. Fig. 8(a) shows the differential normal-incidence reflection from a Ge optical-flat, pumped with $1 \mathrm{GW} \mathrm{cm}^{-2}$ (peak) by the $1064 \mathrm{~nm}$ RegA output of the laser system. The samples are pumped above their saturation intensity, which helps ensure homogenous pumping in the first microns of the material. Differential reflection is defined as the ratio of pumped and un-pumped reflection. The Kolmar-MCT with $\mathrm{LN}_{2}$ cooling was used as the detector. The 0 ps spectrum has
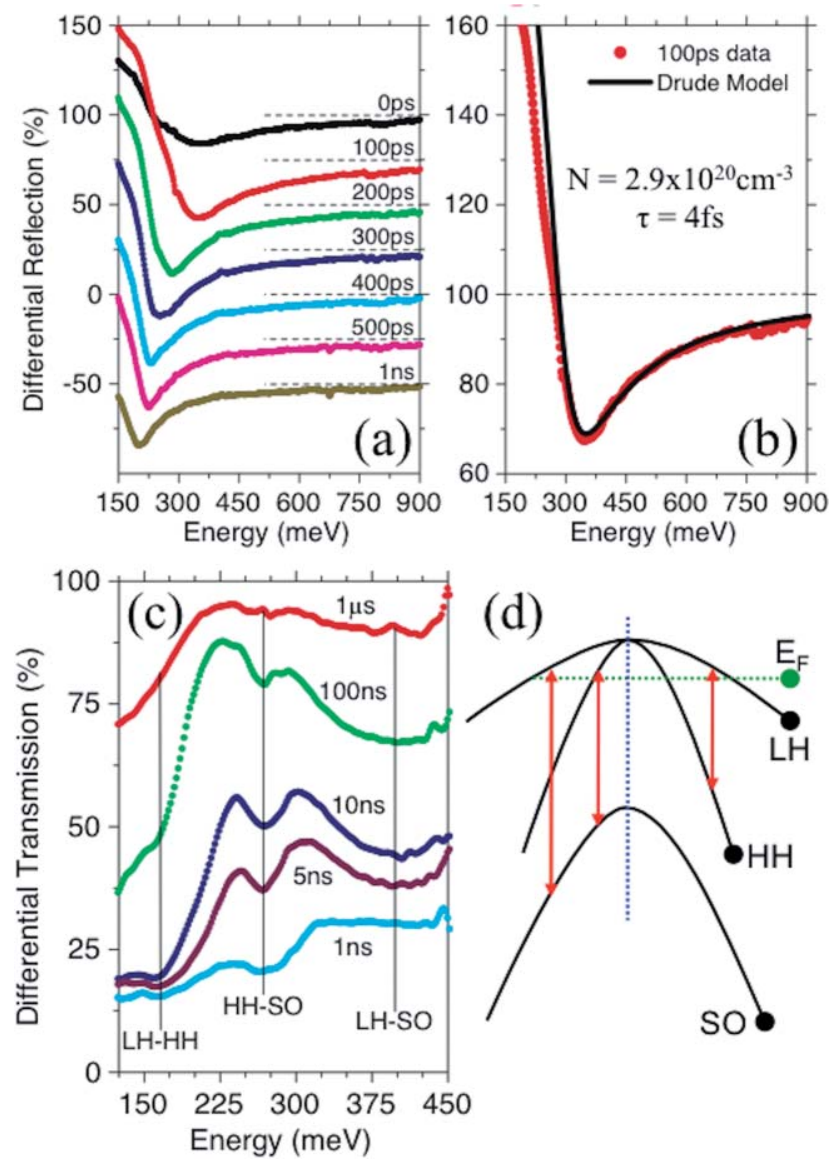

FIG. 8. (Color online) (a) Pumped MIR/NIR differential reflection spectra from a Ge optical-flat, showing a reflectance minimum at the plasma frequency of the injected carriers, from $100 \mathrm{ps}$ to $1 \mathrm{~ns}$. Spectra are shifted vertically for clarity. (b) Shows a fit of the 100 ps data to a simple Drude model giving an optically injected carrier density of $(2.9 \pm 0.5) \times 10^{20} \mathrm{~cm}^{-3}$ and scattering time of $4 \pm 0.5$ fs. (c) Inset shows a fit to a simple Drude model. (c) Differential transmission of the same Ge optical-flat, from $1 \mathrm{~ns}$ to $1 \mu \mathrm{s}$ (three-decades of time). The HH-SO and LH-SO intra-valence band transitions are clearly resolved. (d) Schematic of the valence band-structure in bulk $\mathrm{Ge}$, with $\mathrm{E}_{\mathrm{F}}$ as the quasi-Fermi energy.

a very broad minimum at $350 \mathrm{meV}$, consistent with the high scattering-rate expected during optical pumping. As shown in the $100 \mathrm{ps}$ spectrum, immediately after the pumping, the reflectance minimum narrows considerably. The red-shift in plasma frequency as the carrier recombine is clearly evident over the first $1 \mathrm{~ns}$. A fit of the 100 ps reflectance-spectra, using a simple Drude model (with an effective mass of $0.34 \mathrm{~m}_{\mathrm{e}}$ (Ref. 27)), is shown in Fig. 8(b). Since the carrier density enters this model directly, there is no need to normalize the data to a film-thckness or skin-depth. This fit gives a carrier density of $(2.9 \pm 0.5) \times 10^{20} \mathrm{~cm}^{-3}$ and a scatteringtime of $4 \pm 0.5$ fs. The optically injected carrier density is consistent with the value that calculations indicate are needed for net gain. ${ }^{25}$ The cited measurements on doped Ge (both $n$ and $p$-type) give scattering-times of $\approx 50 \mathrm{fs}$, for densities of the order $10^{19} \mathrm{~cm}^{-3}$. It is not yet known if the significantly lower scattering-time observed during with the IRPP measurement is due to the higher optically injected carrier-density or the elevated carrier temperature during pumping. 
Figure 8(c) shows the differential MIR transmission through the same Ge optical-flat, measured with the Vigo-MCT detector (both transmission and reflection measurements were made at the same time, using the DAQS in dual-channel mode). Here, the origin of the PIA from the strong valence intra-band transitions between the light hole $(\mathrm{LH})$, heavy hole $(\mathrm{HH})$, and split-off $(\mathrm{SO})$ bands $^{28}$ can be investigated. After optical-injection, the valence band becomes populated with holes that can participate in strong "vertical" transitions. A schematic of the valence band-structure and quasi-Fermi energy is shown in Fig. 8(d). Up to $1 \mathrm{~ns}$ after pumping, the MIR transmission is heavily suppressed, but no well-defined spectroscopic features are identifiable. After $1 \mathrm{~ns}$, the HH-SO transition at $270 \mathrm{meV}$ can just be resolved. After $10 \mathrm{~ns}$, it can clearly be seen, and the very broad LH-SO transition at $400 \mathrm{meV}$ has begun to take form. The tail of this strong LH-SO transition decays slowly in energy, and extends to beyond the direct gap, ${ }^{29}$ where it acts to suppress the net optical bleaching. The strong feature below $200 \mathrm{meV}$ in all spectra is attributed to the LH-HH transitions. Note that the differential transmission in this range remains $<75 \%$ even $1 \mu \mathrm{s}$ after pumping. Since both the reflection and transmission measurements were made at the same time, under the same pumping conditions, the magnitude of the valence intra-band absorption can be directly correlated to the carrier concentration (as deduced from the fits to the reflectance minimum).

\section{B. IRPP investigation of Ge-on-Si}

Using the insight into the properties of bulk Ge under strong optical excitation from the previous section, the transmission- and reflection-spectra of a Ge-on-Si system can be interpreted. An analogue of the MIT sample, this Ge-on-Si was prepared by low pressure plasma enhanced chemical vapour deposition of a $1 \mu \mathrm{m}$ Ge-layer directly onto $\mathrm{Si}(100)$ and slightly $n$-type doped with phosphor to 2 $\times 10^{16} \mathrm{~cm}^{-3}$. This sample differs from the MIT material in that it has both a lower tensile strain and doping-level. Full broadband (100-900 meV) spectra from the Ge-on-Si sample were made by compositing spectra taken with the Vigo-MCT and Teledyne-Judson-InSb detectors under exactly the same pumping conditions. The Ge-on-Si sample was also pumped with the RegA output of the laser to approximately $1 \mathrm{GW} \mathrm{cm}^{-2}$. As a control experiment, a sample without the Ge-layer, but with the same Si-substrate was investigated. We did not observe any effect from pumping, either in transmission or reflection spectroscopy. Clearly, absorption of the $1064 \mathrm{~nm}$ of the YAG pump is sufficiently far below the Si band-gap that pump absorption in the substrate is negligible. The spectra in Fig. 9 give both the differential transmission- and reflection-spectra 100 ps after pumping. The $150 \mathrm{meV}$ period oscillation visible on both spectra is due to thin-film interference in the $1 \mu \mathrm{m}$ Ge layer.

To fit the Ge-on-Si reflection data, the simple Drude model must be extended to account for the multiple reflections in the Ge layer. Fitting to the 100 ps data gives a maximum carrier density of $2.5 \pm 0.5 \times 10^{20} \mathrm{~cm}^{-3}$ and a scattering-

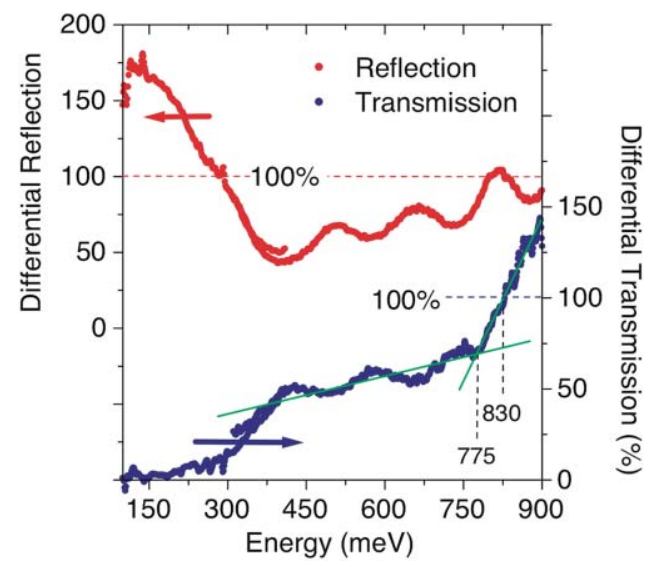

FIG. 9. (Color online) Broadband (100-900 meV) differential transmissionand reflection-spectra of the Ge-on-Si sample, $100 \mathrm{ps}$ after pumping. The spectra are composites of data from the Vigo-MCT and Teledyne-Judson-InSb detectors. Fitting to the Drude model gives an injected carrier density of (2.5 $\pm 0.5) \times 10^{20} \mathrm{~cm}^{-3}$. The strong PIA, extending from the MIR intra-valence band transitions to the direct gap, is clearly evident. As a result of this PIA, the optical bleaching (starting at $775 \mathrm{meV}$ ) only leads to a differential transmission $>100 \%$ for energies greater than $830 \mathrm{meV}$.

time of $2.5 \pm 0.5$ fs. Under the same pumping conditions, both the bulk Ge and Ge-on-Si samples achieve very similar maximum carrier densities, but the scattering-time in the Ge-on-Si sample is significantly shorter. One possible explanation for this observation is a higher carrier temperature in the Ge-on-Si layer due to reduced thermal conductivity to the Si substrate. It should be noted that the deduced carrier density in the Ge-on-Si sample also reaches the level needed for net gain as indicated by calculations. However, as clearly shown by the differential transmission in Fig. 9, a strong PIA from the valence intra-band transitions extends linearly from the LH/HH-SO transitions right up to the direct gap at $775 \mathrm{meV}$. This PIA has a strength of $>3500 \mathrm{~cm}^{-1}$ at the direct gap and acts to heavily suppress the net optical bleaching. As a result, the differential transmission only exceeds $100 \%$ above $830 \mathrm{meV}$, though the optical bleaching clearly begins at $775 \mathrm{meV}$. A PIA of this magnitude effectively prohibits any population inversion from transforming into a net gain. The measurement programme now goes towards investigating Ge-on-Si samples with higher tensile strain and doping.

\section{CONCLUSION}

The newly developed broadband IRPP system provides users with a unique MIR and NIR spectroscopic tool for timeresolved measurements. The widely tuneable $1 \mathrm{kHz}$ optical pump, the broadband SRIR transmitted with all-metal optics, the $100 \mathrm{ps}$ to many-microsecond time-bandwidth and the versatile two-channel DAQS, all combine to give maximum experimental flexibility; opening-up a new approach of "pump anywhere and probe everywhere" in the 1.1-16 $\mu \mathrm{m}$ range. We anticipate that the flexibility of the 100 ps system, both in terms of its spectroscopic range and pump-probe offset times, will be attractive to users from a wide range of fields, including photochemistry, catalysis-studies, and life-sciences. As a showcase, we have used the IRPP system to determine the 
optically injected carrier density, direct gap bleaching, and pump-induced absorption in both bulk Ge and Ge-on-Si systems. These results provide insight into the carrier dynamic, absorption, and inversion effects for the recently reportedly Ge-on-Si laser materials.

\section{ACKNOWLEDGMENTS}

We gratefully acknowledge Dr. Luca Quaroni for X01DC beamline support. The Ge-on-Si sample was prepared by Danny Chrastina, Giovanni Isella, and Hans von Känel (Dipartimento di Fisica del Politecnico di Milano, Italy). Part of this work was funded by the Swiss National Science Foundation (SNSF), contract 200020-116718.

\section{APPENDIX: TUNABLE LASER SYSTEM}

The $100 \mathrm{~Hz}$ bandwidth PLL system is used to synchronize the 100 ps Nd:YAG MO to the SLS. Most of the MO pulse-train then passes through a quasi-CW amplifier stage, but a small fraction is re-directed by a digital pulse-picker to the RegA. The picker passes every 83 520th (must be a multiple of 480 electron "buckets" in the storage-ring) MO pulse to the $1 \mathrm{kHz}$ (83.3 MHz /83 520) RegA. After the amplification stages, there are two $1064 \mathrm{~nm} 100 \mathrm{ps}$ transform-limited pulsetrains; (i) a quasi-CW $83.3 \mathrm{MHz}$ train with average power $=5.3 \mathrm{~W}$, and (ii) the RegA $1 \mathrm{kHz}$ train with average power $=2.8 \mathrm{~W}$. The quasi-CW train drives a synchronously pumped OPO. $^{21}$

The OPO consists of a temperature-tuned, multi-element periodically poled lithium niobate (PPLN) crystal as the active material, and a blazed diffraction grating output-coupler as the wavelength selection element. Both PPLN temperature and grating position are electronically controlled, allowing for remote control of OPO tuning. The OPO produces two quasi$\mathrm{CW}$ trains, (i) OPO-signal (1.55-2.02 $\mu \mathrm{m}$ ), and (ii) OPO-idler $(2.25-3.35 \mu \mathrm{m})$ at $83.3 \mathrm{MHz}$. The wavelength of the signal and idler outputs are correlated through energy conservation $\left(1 / \lambda_{\text {signal }}+1 / \lambda_{\text {idler }}=1 / \lambda_{\text {Nd:YAG }}\right)$, and the user-defined grating condition. The OPO-signal train seeds an OPA, which is synchronously driven by the $1064 \mathrm{~nm}$ RegA $1 \mathrm{kHz}$ train. ${ }^{22}$ To span its full tuning range, the OPA uses two pairs of angletuned KTA crystals. It produces near-transform-limited OPAsignal and OPA-idler trains at $1 \mathrm{kHz}$. Crystal sets KTA(1) and KTA(2) generate OPA-signal from $1.55-1.65 \mu \mathrm{m}$ and 1.65 $-2.02 \mu \mathrm{m}$, respectively. The OPA-signal and OPA-idler are orthogonally polarized, and so can be conveniently separated using a Rochon polarizer.

The tuning range of the laser system is extended towards the MIR by performing difference frequency generation (DFG) between the OPA-signal and OPA-idler trains. The mixing is carried-out in an angle-tuned GaSe crystal. ${ }^{23}$ The DFG tuning range is $3.5-16 \mu \mathrm{m}$, with a wavelength given by $\left(1 / \lambda_{\mathrm{DFG}}=1 / \lambda_{\text {signal }}-1 / \lambda_{\text {idler }}\right)$, or equivalently by $\left(1 / \lambda_{\mathrm{DFG}}\right.$ $\left.=1 / \lambda_{\mathrm{Nd} \text { :YAG }}-2 / \lambda_{\text {idler }}\right)$. The tuning range of the system is extended below the OPA-signal limit of $1.55 \mu \mathrm{m}$, through second harmonic generation (SHG) from the OPA-idler train. An external, single-pass SHG geometry, consisting of a third pair of angle-tuned KTA crystals, is used. The SHG-idler tuning range is $1.1-1.5 \mu \mathrm{m}$. For every process (excluding the crystal set swap of $\operatorname{KTA}(1)$ and $\operatorname{KTA}(2)$, all angle and temperature tuning of the optical elements is computer controlled, offering users fully programmable "hands free" tuning of the laser-system. Unifying the OPA, DFG, and SHG outputs to the same optical axis (from their separate output-ports on the laser-system) is achieved using several flipping mirrors and a motorized translation stage. To aid alignment, a low-power visible diode-laser is also aligned with respect to the optical axis.

${ }^{1}$ D. H. Bilderback, P. Elleaume, and E. Weckert, J. Phys. B 38, S773 (2005).

${ }^{2}$ W. D. Duncan and G. P. Williams, Appl. Opt. 22(18), 2914 (1983).

${ }^{3}$ J. Yarwood, T. Shuttleworth, J. B. Hasted, and T. Nanba, Nature (London) 312, 742 (1984)

${ }^{4}$ T. Naba, Rev. Sci. Instrum. 60, 1680 (1989)

${ }^{5}$ G. P. Williams et al., Rev. Sci. Instrum. 60, 2178 (1989).

${ }^{6}$ J. A. Reffner, P. A. Martoglio, and G. P. Williams, Rev. Sci. Instrum. 66, 1298 (1995)

${ }^{7}$ G. L. Carr, J. A. Reffner, and G. P. Williams, Rev. Sci. Instrum. 66, 1490 (1995).

${ }^{8}$ M. C. Martin, U. Schade, P. Lerch, and P. Dumas, Trends Anal. Chem. 29(6) 453 (2010).

${ }^{9}$ G. L. Carr, R. P. S. M. Lobo, J. LaVeigne, D. H. Reitze, and D. B. Tanner, Phys. Rev. Lett. 85(14), 3001 (2000).

${ }^{10}$ R. P. S. M. Lobo, J. D. LaVeigne, D. H. Reitze, D. B. Tanner, and G. L. Carr, Rev. Sci. Instrum. 73, 1 (2002).

${ }^{11}$ G. L. Carr, R. P. S. M. Lobo, C. J. Hirschmug, J. LaVeigne, D. H. Reitze, and D. B. Tanner, Proc. SPIE 3153, 80 (1997).

${ }^{12}$ Y. Tamaki, A. Furube, M. Murai, K. Hara, R. Katoh, and M. Tachiya, J. Am.Chem. Soc. 128, 416 (2006).

${ }^{13}$ R. Brudler, R. Rammelsberg, T. T. Woo, E. D. Getzoff, and K. Gerwert, Nat. Struct. Biol. 8, 265 (2001).

${ }^{14}$ M. Hein, A. A. Wegener, M. Engelhard, and F. Siebert, Biophys. J. 84, 1208 (2003).

${ }^{15}$ X. Z. Sun, S. M. Nikiforov, J. Yang, C. S. Colley, and M. W. George, Appl. Spectrosc. 56, 31 (2002).

${ }^{16}$ W. Hage, M. Kim, H. Frei, and R. A. Mathies, J. Phys. Chem. 100, 16026 (1996).

${ }^{17}$ G. D. Smith and R. A. Palmer, "Fast time-resolved mid-infrared spectroscopy using an interferometer," in Handbook of Vibrational Spectroscopy, edited by J. M. Chalmers and P. R. Griffiths, (Wiley, New York, 2002), Vol. 1, p. 625

${ }^{18}$ J. Liu, X. Sun, R. Camacho-Aguilera, L. Kimerling, and J. Michel, Opt. Lett. 35(5), 679 (2010).

${ }^{19} \mathrm{See}$ http://www.psi.ch/sls/ir/ for the home-page of the X01DC infrared beam-line of the SLS, containing technical specifications of different available end-stations.

${ }^{20}$ We have not yet accessed the full $\times 100$ brilliance advantage, because of losses in the transfer line, image errors, and a coupling-scheme into the FTIR that was optimized for micro-spectroscopy work.

${ }^{21}$ K. Finsterbusch, R. Urschel, and H. Zacharias, Appl. Phys. B 70, 741 (2000).

${ }^{22}$ K. Finsterbusch, R. Urschel, and H. Zacharias, Appl. Phys. B 74, 319 (2002).

${ }^{23}$ K. Finsterbusch, A. Bayer, and H. Zacharias, Appl. Phys. B 79, 457 (2004).

${ }^{24}$ T. Scarvie, N. Andresen, K. Baptiste, J. Byrd, M. Chin, M. C. Martin, W. R. McKinney, and C. Steier, Infrared Phys. Technol. 45, 403 (2004).

${ }^{25}$ J. Liu, X. Sun, D. Pan, X. Wang, L. C. Kimerling, T. L. Koch, and J. Michel, Opt. Express 15(18), 11272 (2007).

${ }^{26}$ W. Spitzer and H. Fan, Phys. Rev. 106(5), 882 (1957).

${ }^{27}$ H. Y. Fan, Rep. Prog. Phys. 19, 107 (1956).

${ }^{28}$ W. Kaiser, R. Collins, and H. Fan, Phys. Rev. 91(6), 1380 (1953).

${ }^{29}$ H. Briggs and R. Fletcher, Phys. Rev. 91(6), 1342 (1953). 